\title{
Longitudinal EEG Studies in a Kindred with Lafora Disease
}

\author{
C. Yen, A. Beydoun, and I. Drury \\ Department of Neurology, University of Michigan, Ann Arbor, Michigan, U.S.A.
}

\begin{abstract}
Summary: We reviewed 18 EEG studies in four members of a family with the Lafora form of progressive myoclonic epilepsy. Each patient was the product of a consanguinous marriage and presented as a teenager with progressive seizures, myoclonus, dementia, and ataxia, and had biopsy proven disease. The EEG early in Lafora disease has spike-wave activity resembling that seen in a primary generalized epilepsy; the background slowing is more typical of a secondary generalized epilepsy. With
\end{abstract}

disease progression, there is increased epileptiform activity, and a striking change in the spike-wave complexes, with a marked increase in frequency up to $6-12 \mathrm{~Hz}$, and many more short duration polyspike components. Unlike some other forms of secondarily generalized epilepsy, the EEG in Lafora disease is distinguished by an increased frequency of the spike-wave complexes with disease progression. Key Words: Lafora disease-SeizuresElectroencephalography-Genetics.
Lafora disease (LD) is a form of progressive myoclonic epilepsy, characterized by seizures, myoclonus, dementia, and ataxia, with onset in adolescence and progression to death within several years (Van Heycop Ten Ham, 1968; Schwarz, 1977). Most descriptions of the electroencephalogram (EEG) in LD have been isolated case reports. One series (Tassinari et al., 1978) found early EEG changes similar to those in primary generalized epilepsy, but with progression the development of background slowing, polyspikes, and diffuse and multifocal spikes. We report the longitudinal EEG findings in four members of a kindred with LD. These patients had significant EEG findings that made discrimination from a primary epilepsy possible in the early stage of illness. With progression, all developed fast-frequency spike-waves of $10 \mathrm{~Hz}$ or greater, an unusual feature that may be quite distinctive in this disorder.

Received July 1990; revision accepted October 1990.

Address correspondence and reprint requests to Dr. 1. Drury at EEG Laboratory, University of Michigan Medical Center, 1500 E. Medical Center Drive, Ann Arbor, MI 48109-0036, U.S.A.

This work was presented in part at the 1990 Annual Meeting of the Central Association of Electroencephalographers and published as an abstract in Electroencephalogr Clin Neurophysiol $1991 ; 78: 14$ p.

\section{MATERIALS AND METHODS}

All four children were products of consanguinous marriages; two were brother and sister, and the others were first cousins. Each had the typical clinical features and progressive course of LD, with diagnosis confirmed by a skin or brain biopsy. Followup has been from 2.5 to 9 years; three patients have died. The principal clinical features are summarized in Table 1. Two illustrative case histories follow.

\section{Patient \#1}

An isolated generalized tonic-clonic seizure occurred at age 12 years in this girl with normal birth and developmental history. In the following year, she developed severe behavioral problems, dysarthria, and ataxia, as well as poorly controlled absence, myoclonic, and tonic-clonic seizures. She became bedridden 4 years after onset. A corpus callosotomy temporarily decreased the seizure frequency; the diagnosis of LD was made at that time by a brain biopsy. The patient died 8 years after onset from multiple episodes of aspiration pneumonia.

\section{Patient \#4}

At age 14 years, this girl developed myoclonic jerks of the extremities, but was still doing well in school. Birth and developmental history were normal. A brother had LD. Treatment with valproate 
TABLE 1. Clinical features in kindred with Lafora disease

\begin{tabular}{|c|c|c|c|c|c|c|}
\hline $\begin{array}{l}\text { Patient } \\
\text { number }\end{array}$ & Sex & $\begin{array}{c}\text { Age at } \\
\text { onset } \\
\text { (years) }\end{array}$ & $\begin{array}{l}\text { Presenting } \\
\text { symptoms }\end{array}$ & $\begin{array}{l}\text { Seizure } \\
\text { type }\end{array}$ & $\begin{array}{l}\text { Years } \\
\text { followed }\end{array}$ & $\begin{array}{c}\text { Number of } \\
\text { electroencephalograms }\end{array}$ \\
\hline $1^{\bar{a}}$ & $\mathrm{~F}$ & 12 & Seizure & $\begin{array}{l}\text { Tonic-clonic, } \\
\text { myoclonic, absence }\end{array}$ & 8 & 7 \\
\hline $2^{a}$ & $\mathrm{~F}$ & 15 & Myoclonus & $\begin{array}{l}\text { Myoclonic, } \\
\text { tonic-clonic, absence }\end{array}$ & 9 & 2 \\
\hline $3^{a}$ & $\mathbf{M}$ & 15 & Seizure & Tonic-clonic, myoclonic & 5 & 7 \\
\hline 4 & $\mathrm{~F}$ & 14 & Myoclonus & Myoclonic, tonic-clonic & 2.5 & 2 \\
\hline
\end{tabular}

${ }^{a}$ Deceased.

resulted in initial improvement. Over several months, school performance deteriorated and generalized tonic-clonic seizures developed. Ataxia, dementia, and myoclonus have progressed but she is still ambulatory 2.5 years after onset.

Each patient had an initial EEG within 1 year of symptom onset and at least one subsequent EEG. A total of 18 studies were performed (range of two to seven) using the International 10-20 system of electrode placement on 8- to 21-channel instruments, with hyperventilation and photic stimulation performed routinely. All were obtained in the waking and drowsy states. Each was reviewed for background frequency and reactivity; the morphology, location, and frequency of the spike-wave activity; response to activating procedures; and the presence of independent focal spikes especially in the occipital regions. EEGs were separated according to stage of illness defined as early $(<1$ year from onset), intermediate (1-3 years from onset), or late $(>3$ years from onset).

\section{RESULTS}

The EEG features are summarized in Table 2. In the early stage (Fig. 1), there was mild slowing of the background activity that was reactive to eye opening in only one case. Trains of irregular, bilaterally synchronous $3 \mathrm{~Hz}$ spike-wave activity were seen at times diffusely, and at other times with an anterior or posterior emphasis. Two patients had EEGs performed in the intermediate stage. Both had increased background slowing (Fig. 2) and in one the frequency of the spike-waves had increased. In the late stage (Fig. 3), there was severe background slowing and a marked increase in the amount of epileptiform activity. In all cases, this consisted predominantly of $6-12 \mathrm{~Hz}$ spike-waves and short duration polyspikes, generally synchronous, with a diffuse or posterior emphasis. All patients had both synchronous and asynchronous epileptiform activity in the occipital areas. We were unable to reliably discriminate independent occipital spikes from fragments of the generalized discharges.

A photoparoxysmal response was seen inconsistently in three patients, although one had a brief generalized myoclonic seizure induced by photic stimulation. Hyperventilation was rarely activating. Myoclonus was also seen, frequently with no electrographic accompaniment.

TABLE 2. Electroencephalographic features in Lafora disease

\begin{tabular}{|c|c|c|c|c|c|c|c|c|}
\hline \multirow{3}{*}{$\begin{array}{l}\text { Patient } \\
\text { number }\end{array}$} & \multirow{3}{*}{$\begin{array}{l}\text { Stage of } \\
\text { illness }\end{array}$} & \multicolumn{2}{|c|}{ Background } & \multicolumn{3}{|c|}{ Spike-wave activity } & \multirow{2}{*}{\multicolumn{2}{|c|}{ Activation }} \\
\hline & & \multirow{2}{*}{$\begin{array}{l}\text { Predominant } \\
\text { frequency }\end{array}$} & \multirow[b]{2}{*}{ Reactivity } & \multirow[b]{2}{*}{ Morphology } & \multirow[b]{2}{*}{ Location } & \multirow{2}{*}{$\begin{array}{l}\text { Predominant } \\
\text { frequency }\end{array}$} & & \\
\hline & & & & & & & $\overline{\mathrm{Hv}}$ & Photic \\
\hline \multirow[t]{3}{*}{1} & Early & 7 & - & Single $\mathbf{S}-\mathbf{W}$ & Diffuse & 3 & + & + \\
\hline & Intermediate & 4 & - & Single $S-W$ & Posterior & 3 & - & - \\
\hline & Late & $2-3$ & - & $\begin{array}{l}\text { Single and } \\
\text { poly } S-W\end{array}$ & Posterior & $8-10$ & ND & - \\
\hline \multirow[t]{2}{*}{2} & Early & $4-7$ & - & Single $S-W$ & Posterior & 3 & - & - \\
\hline & Late & $3-5$ & - & $\begin{array}{l}\text { Single and } \\
\text { poly } S-W\end{array}$ & Posterior & $6-8$ & ND & + \\
\hline \multirow[t]{2}{*}{3} & Early & 4 & - & Single $\mathrm{S}-\mathrm{W}$ & Diffuse & 3 & - & - \\
\hline & Late & $2-3$ & - & $\begin{array}{l}\text { Single and } \\
\text { poly } S-W\end{array}$ & Diffuse & $8-10$ & ND & - \\
\hline \multirow[t]{2}{*}{4} & Early & 6 & + & Single S-W & Anterior & 3 & - & + \\
\hline & Intermediate & 4 & + & $\begin{array}{l}\text { Single and } \\
\text { poly } S-W\end{array}$ & Diffuse & 10 & - & - \\
\hline
\end{tabular}

ND, not done; Hv, hyperventilation; S-W, spike-wave; Hz, Hertz. 


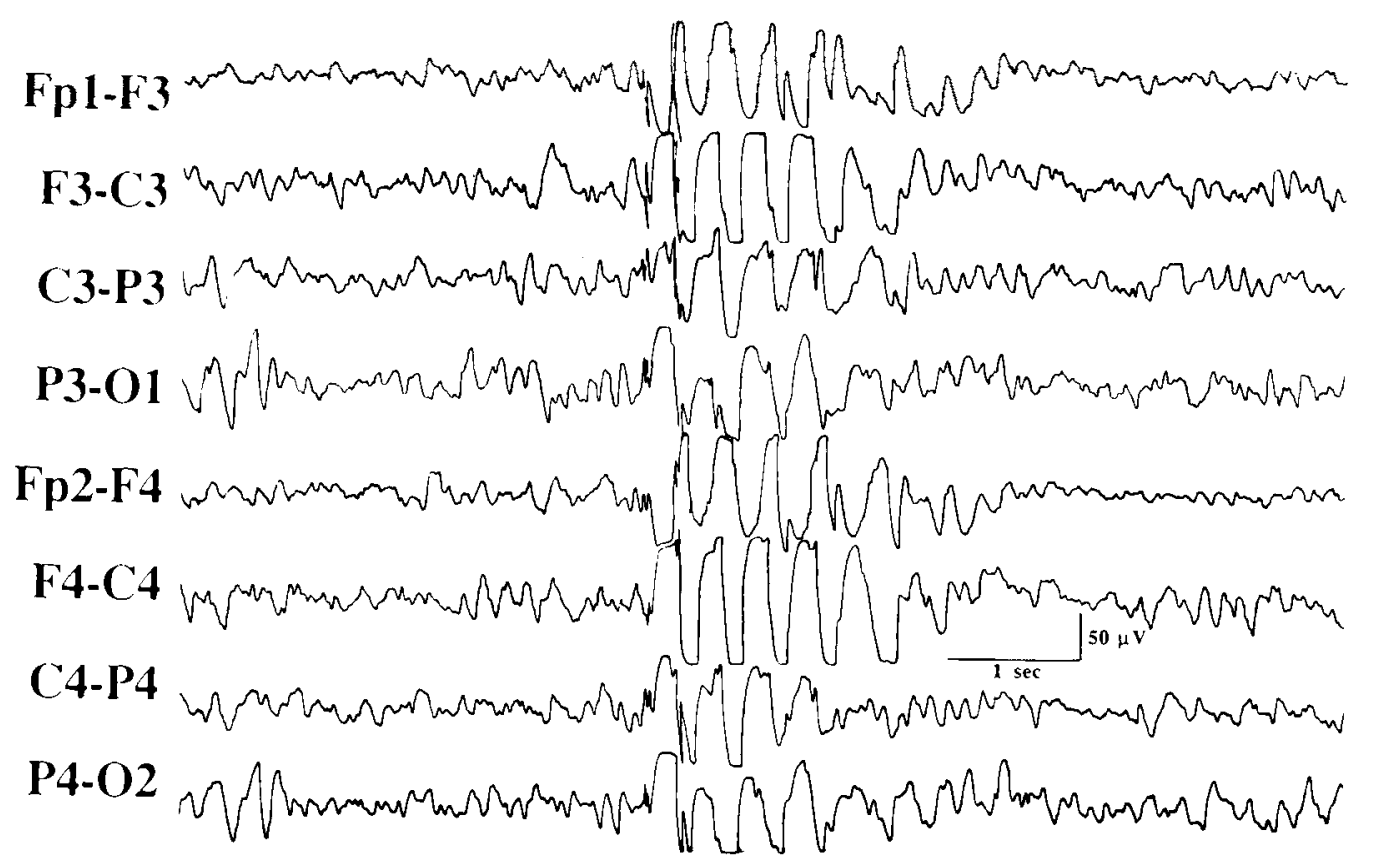

FIG. 1. Patient \#1. Early stage, age 12 years, 1 week after first seizure. Electroencephalogram shows mild background slowing and trains of $3 \mathrm{~Hz}$ irregular spike-wave activity.

\section{DISCUSSION}

The progressive myoclonic epilepsies constitute one subtype of patients with secondary generalized epilepsy (Berkovic et al., 1986), and are a heterogenous group that can be distinguished by clinical features, biopsy findings, and in some patients by particular EEG characteristics. In some early case reports of LD (Van Heycop Ten Ham and De Jager, 1963; Janeway et al., 1967), the EEG pattern was thought to be nonspecific and nonprogressive, with background slowing and both diffuse and focal epileptiform activity. In a later series, Tassinari et al (1978) described an early stage (first year) of LD with EEG features similar to those seen in primary generalized epilepsy, with normal background activity and in four of six cases $3-5 \mathrm{~Hz}$ polyspike-andwave activity anteriorly. None of our cases demonstrated a normal background, although all had an EEG within 1 year of onset; patient 1 was evaluated within a week of first seizure, with no medication, and the EEG already had mild slowing. Progressive

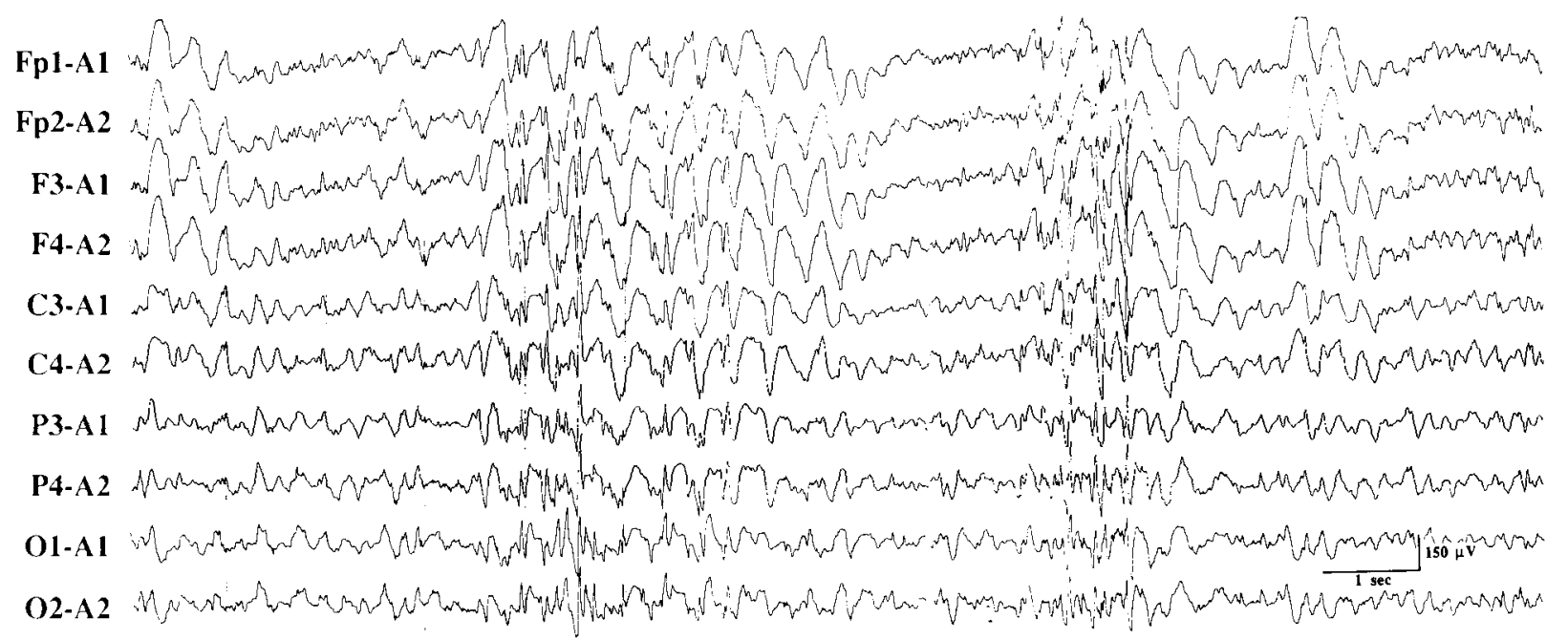

FIG. 2. Patient \#4. Intermediate stage, age 16 years, 2 years after onset. Electroencephalogram shows remnants of $3 \mathrm{~Hz}$ spike-waves, with faster frequency spike-waves up to $12 \mathrm{~Hz}$. 


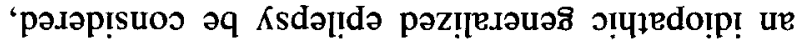

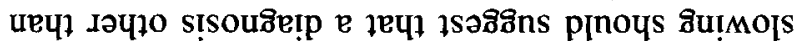

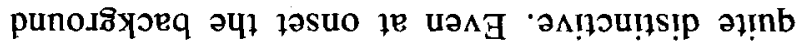

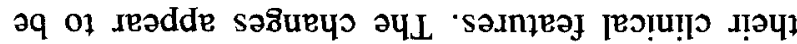

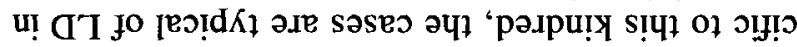

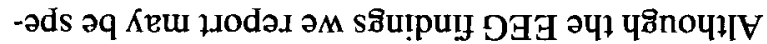

uolissajo

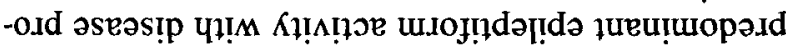

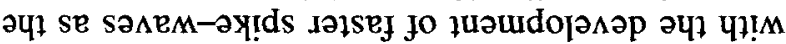

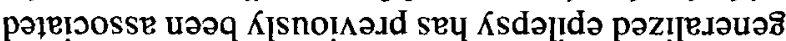

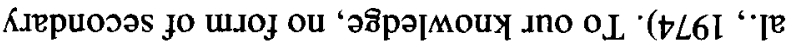

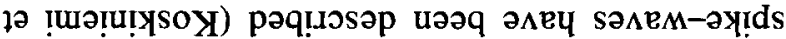

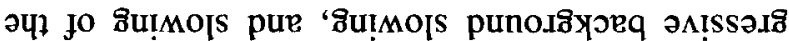

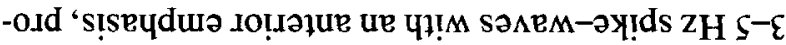

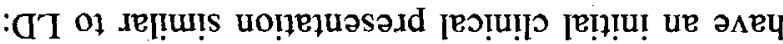

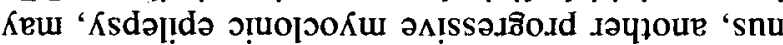

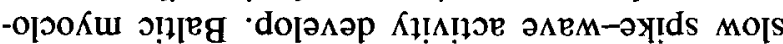
pur 8u!Mo[s punosôy

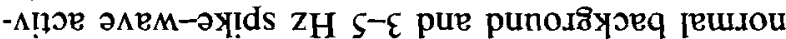

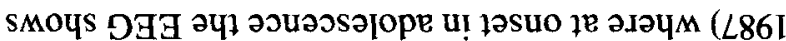

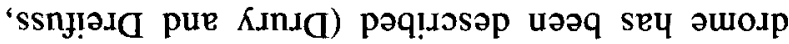

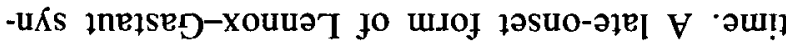

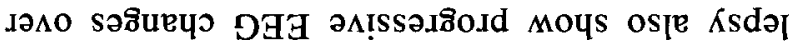

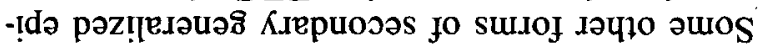

'saริseyวsip

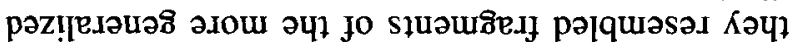

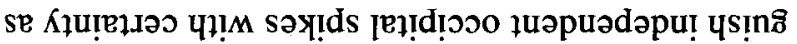
-u!̣stp ou pinos ә

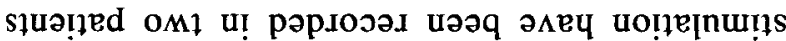

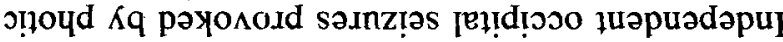

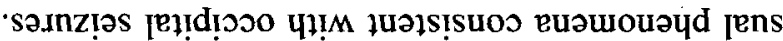

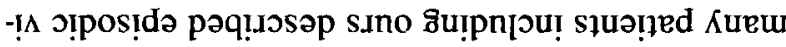

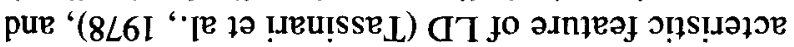

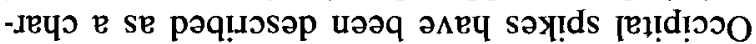

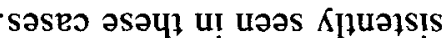

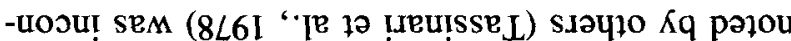

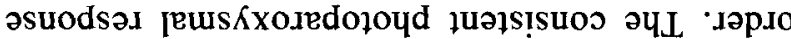

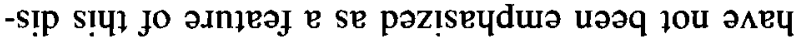

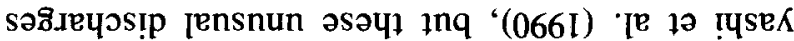

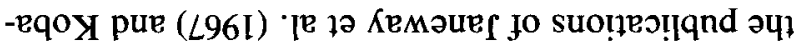

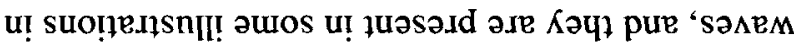

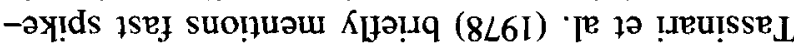
- KI!

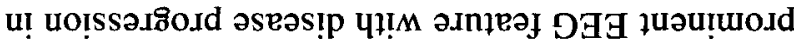

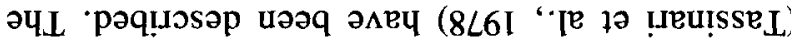

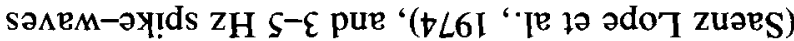

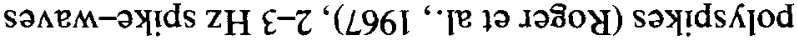

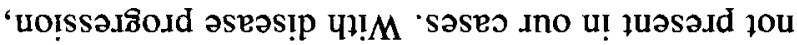

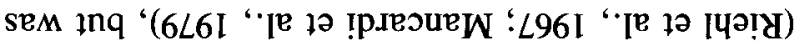

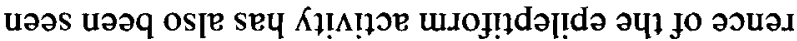
-snoal э!po!ıә

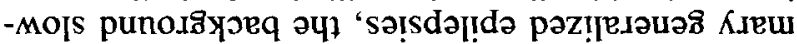
-!Id u! uəว

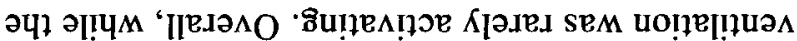

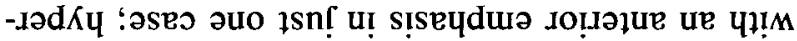

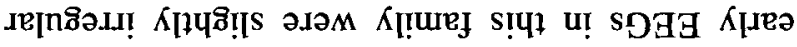

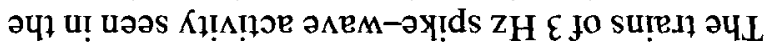

-səuidə

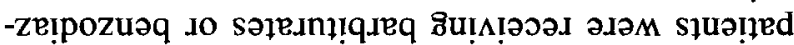

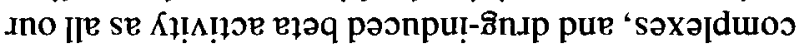

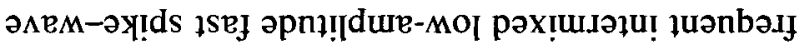

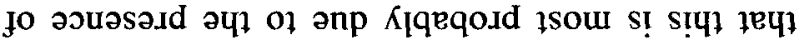

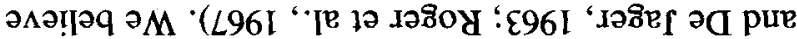

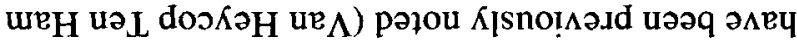

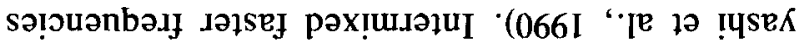

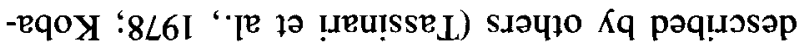

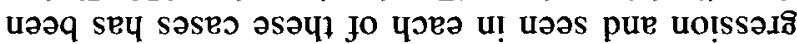

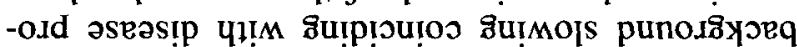

'say!dsא|Od pue sanem-ax!ds $15 e_{1}$

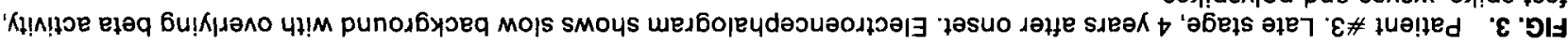

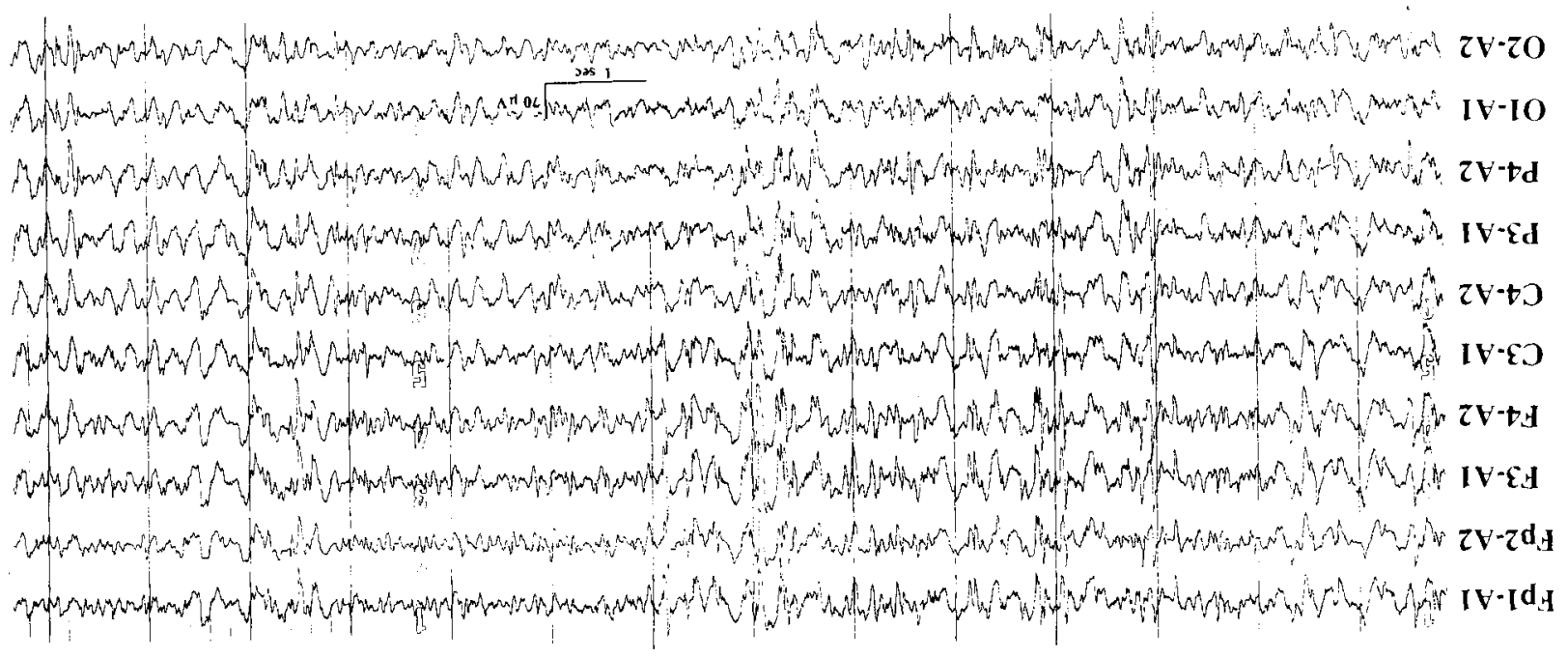


and the progression to prominent fast spike-wave complexes over time appears to be unique.

\section{REFERENCES}

Berkovic SF, Andermann F, Carpenter S, et al. Progressive myoclonus epilepsies: specific causes and diagnosis. $N$ Engl $J$ Med 1986;315:296-305.

Drury I, Dreifuss FE. Late-onset Lennox-Gastaut syndrome. In: Wolf P, Dam M, Janz D, Driefuss FE, eds. Advances in epileptology, l6th Epilepsy International symposium. New York: Raven Press, 1987:211-6.

Janeway R, Ravens JR, Pearce LA, et al. Progressive myoclonus epilepsy with Lafora inclusion bodies. I. Clinical, genetic, histopathologic and biochemical aspects. Arch Neurol 1967;16:565-82.

Kobayashi K, Iyoda K, Ohtsuka $\mathrm{Y}$, et al. Longitudinal clinicoelectrophysiologic study of a case of Lafora disease proven by skin biopsy. Epilepsia 1990;31:194-201.

Koskiniemi M, Toivakka E, Donner M. Progressive myoclonus epilepsy. Electroencephalographical findings. Acta Neurol Scand 1974;50:333-59.

Mancardi GL, Primavera A, Leonardi A, et al. Tendency to periodic recurrence of EEG changes in Lafora's disease. Case report. Eur Neurol 1979;18:129-35.

Riehl J, Lee DK, Andrews JM, et al. Electrophysiological and neuropharmacological studies in a patient with UnverrichtLafora's disease. Neurology 1967;17:502-11.

Roger J, Gastaut H, Boudouresques J, et al. Epilepsie-myoclonie progressive avec corps de Lafora. Étude clinique et polygraphique controle anatomique ultra-structural. Rev Neurol 1967;116:197-212.

Saenz Lope E, Ramon y Cajal Junquera S, Berenguel AB. Progressive myoclonic epilepsy with Lafora's bodies. A clinicopathological study. Acta Neurol Scand 1974;50:537-52.

Schwarz GA. Lafora's disease: a disorder of carbohydrate metabolism. In: Goldensohn ES, Appel SH, eds. Scientific approaches to clinical neurology. Philadelphia: Lea \& Febiger, 1977:148-59.

Tassinari CA, Bureau-Paillas M, Bernardina BD, et al. La maladie de Lafora. Rev Electroencephalogr Neurophysiol 1978;8:107-22.

Tinuper P, Aguglia U, Pellissier JF, et al. Visual ictal phenomena in a case of Lafora disease proven by skin biopsy. Epilepsia $1983 ; 24: 214-8$.

Tinuper P, Gobbi G, Aguglia U, et al. Occipital seizures in Lafora disease: a further case documented by EEG. Clin Electroencephalogr 1985;16:167-70.

Van Heycop Ten Ham MW. Lafora disease. A form of progressive myoclonus epilepsy. In: Vinken PJ, Bruyn GW, eds. Handbook of clinical neurology, Vol. 15. New York: American Elsevier, 1968:382-422.

Van Heycop Ten Ham MW, De Jager H. Progressive myoclonus epilepsy with Lafora bodies. Clinical-pathological features. Epilepsia 1963;4:95-119.

\section{RÉSUMÉ}

Les auteurs ont revu 18 enregistrements EEG pratiqués chez 4 membres d'une famille présentant une maladie de Lafora.
Chaque patient était issu d'un mariage consanguin et a présenté dans l'adolescence l'association progressive de crises, d'un myoclonus, d'une démence et d'une ataxie; le diagnostic de la maladie a été confirmé par une biopsie. L'EEG présente, à la phase initiale de la maladie de Lafora, un aspect comparable à celui de l'épilepsie généralisée idiopathique, mais le ralentissement de l'activité de fond évoque davantage l'EEG des épilepsies généralisées symptomatiques (EGS). Pendant la progression de la maladie, les auteurs ont constaté une augmentation de l'activité épileptique EEG, avec modification importante de la morphologie des PO, qui ont augmenté de fréquence, jusqu'à $6-12 \mathrm{c} / \mathrm{s}$, avec composantes accrues en Polypointes rapides. Contrairement à d'autres formes d'EGS, l'EEG de la maladie de Lafora se distingue par une augmentation de fréquence des PO au cours de l'évolution.

(P. Genton, Marseille)

\section{RESUMEN}

Se revisan 18 estudios de EEG en 4 miembros de una familia con la forma de Lafora de una epilepsía mioclónica progresiva. Cada paciente fue producto de un matrimonio consanguíneo y en su adolescencia presentaron ataques progresivos, mioclonías, demencia y ataxia, y se les practicó una biopsia que confirmó la enfermedad. Los EEGs practicados precozmente en la enfermedad de Lafora tienen una actividad de punta-onda que semeja la que se observa en epilepsía generalizada primaria y la lentificación de la actividad de fondo es más típica de epilepsía generalizada sencundaria. A medida que la enfermedad progresa se observó un incremento de la actividad epileptiforme y un cambio sorprendente en los complejos punta-onda con marcado incremento de la frecuencia hasta 6-12 Hz. y muchos más componentes de polipuntas de breve duración. Contrariamente a otras formas de epilepsía generalizada secundaria el EEG en la enfermedad de Lafora se caracteriza por un incremento de la frecuencia de los complejos punta-onda a medida que la enfermedad progresa.

(A. Portera-Sánchez, Madrid)

\section{ZUSAMMENFASSUNG}

Wir beurteilten 18 EEG-Untersuchungen bei 4 Mitgliedern einer Familie mit der Lafora-Form der progressiven MyoklonusEpilepsie. Jeder Patient entstammte einer konsanguinen Ehe und wurde als Teenager mit progressiven Anfällen, Myoklonus, Demenz und Ataxie auffällig; die Diagnose wurde durch Biopsie bestätigt. Das EEG im frühen Verlauf einer Lafora-Erkrankung zeigt Spike Wave Aktivität, die der bei primär generalisierter Epilepsie ähnelt, während die Hintergrundaktivität mehr für eine sekundär generaliserte Epilepsie typisch ist. Mit fortschreitender Erkrankung tritt vermehrt epileptische Aktivität und ein deutlicher Wechsel der Spike Wave Komplexe mit starkem Ansteigen der Frequenz auf 6-12 $\mathrm{Hz}$ und vermehrten kurzdauernden Polyspike-Komponenten auf. Im Gegensatz zu anderen Formen sekundär generalisierter Epilepsien zeigt das EEG bei der Lafora-Erkrankung mit Erkrankungsfortgang eine zunehmende Frequenz der Spike Wave Komplexe.

(C. K. Benninger, Heidelberg) 